\title{
TESTLoc: protein subcellular localization prediction from EST data
}

\author{
Yao-Qing Shen", Gertraud Burger
}

\begin{abstract}
Background: The eukaryotic cell has an intricate architecture with compartments and substructures dedicated to particular biological processes. Knowing the subcellular location of proteins not only indicates how bio-processes are organized in different cellular compartments, but also contributes to unravelling the function of individual proteins. Computational localization prediction is possible based on sequence information alone, and has been successfully applied to proteins from virtually all subcellular compartments and all domains of life. However, we realized that current prediction tools do not perform well on partial protein sequences such as those inferred from Expressed Sequence Tag (EST) data, limiting the exploitation of the large and taxonomically most comprehensive body of sequence information from eukaryotes.

Results: We developed a new predictor, TESTLoc, suited for subcellular localization prediction of proteins based on their partial sequence conceptually translated from ESTs (EST-peptides). Support Vector Machine (SVM) is used as computational method and EST-peptides are represented by different features such as amino acid composition and physicochemical properties. When TESTLoc was applied to the most challenging test case (plant data), it yielded high accuracy ( $85 \%)$.

Conclusions: TESTLOC is a localization prediction tool tailored for EST data. It provides a variety of models for the users to choose from, and is available for download at http://megasun.bch.umontreal.ca/ shenyq/TESTLoc/TESTLoc. html
\end{abstract}

\section{Background}

In eukaryotes, the majority of proteins are encoded in the nuclear genome and translated on ribosomes in the cytosol. Proteins are then transported to different subcellular locations, such as the nucleus, mitochondria, chloroplasts, peroxisomes, etc., where they perform their particular roles in various biological processes. Knowledge of subcellular location is an important asset in the annotation of newly discovered proteins, as it bears clues about a protein's function. Further, knowing the location of proteins and their molecular function allows us to infer where in the cell the corresponding biological process takes place, what the physiological role of this process may be, and how the various processes are spatially integrated. Finally, information on the makeup of proteomes from bacteria-derived organelles (mitochondria and

\footnotetext{
* Correspondence: yaoqing.shen@umontreal.ca
Robert-Cedergren Center for Bioinformatics and Genomics; Biochemistry

* Correspondence: yaoging.shen@umontreal.ca Department, Université de Montréal, 2900 Edouard-Montpetit, Montreal, QC, H3T 1J4, Canada
}

(c) 2010 Shen and Burger; licensee BioMed Central Ltd. This is an Open Access article distributed under the terms of the Creative Commons Attribution License (http://creativecommons.org/licenses/by/2.0), which permits unrestricted use, distribution, and reproduction in any medium, provided the original work is properly cited. chloroplasts) helps to elucidate the migration of proteincoding genes from the endosymbiont to the host.

A variety of experimental approaches are available today for identifying the subcellular localization of proteins, for example, co-expression of fluorescent proteins $[1,2]$, immunofluorescence labeling [3], gene knockout/ knockdown [4], and proteomics techniques such as liquid-chromatography-tandem mass spectrometry (LCMS/MS) [5,6]. However, for most species, large-scale experimental identification of protein subcellular localization remains too expensive or unfeasible. This has set the stage for bioinformatics approaches to predict localization in silico.

Can localization of a protein be confidently inferred via finding a homolog of known location by BLAST [7]? A previous study indicated that localization can be predicted with up to $90 \%$ accuracy when BLAST identity is $50 \%$ or more, but that it falls short for more distant sequences (e.g., only 50\% accuracy for $20 \%$ local identity, Additional file 1) [8]. Further, this approach ignores 
established biological knowledge that homologous proteins are not necessarily located in the same cellular compartment. For example, homologous beta oxidation enzymes are targeted to mitochondria in human and peroxisomes in yeast [9]. Most importantly, the BLAST approach fails for divergent and novel proteins as they do not find significant matches in databases (see Additional file 1). For all these reasons, the bioinformatics community turned to more suited approaches for protein localization prediction.

Today, more than 20 dedicated tools are available for in silico protein localization prediction based on annotation or solely the sequence of proteins (Additional file 2). Annotation information includes textual description taken from the SWISSPROT database, the Gene Ontology database, or PubMed literature [10-12]. Also used for localization prediction is co-occurrence of functional motifs or structural domains in proteins $[13,14]$. Sequence-based tools recognize specific targeting signals that guide proteins to different cellular compartments [15-19]. Alternatively, proteins are classified according to single amino acid frequency $[20,21]$, dipeptide and gapped amino acid pair composition [22-25], or physicochemical properties of amino acids [26]. More recently published predictors combine different protein features [27-31], or integrate annotation with sequences-based prediction $[32,33]$. Finally, meta-predictors combine predictions from several heterogeneous tools [34-36].

Two recent studies evaluated the performance of available localization predictors using datasets that contain only sequences not included in, nor similar to, those in the training sets of these predictors $[37,38]$. One identified as best performing tools BaCelLo [39], LOCtree [29], Protein Prowler [18], TargetP [16], and Wolf-PSORT [40], and the other evaluated BaCelLo, YLoc [38], MuitiLoc2 [32], and KnowPred [41] as best (for sequence features and computational methods used, see Additional file 2). In general, these tools have lower performance on data from plants compared to nonphotosynthetic organisms such as animals and fungi, and this is due to the presence of mitochondria plus chloroplasts in the cell of plants. Both organelles descend from endosymbiotic bacteria and have their own machineries for protein import, DNA replication, and gene expression. This makes it difficult for the tools to distinguish the proteins from the two organelles.

In silico localization prediction tools use full-length protein sequences that are usually inferred from genome sequence. Yet, for many eukaryotic groups of interest are only EST (Expressed Sequence Tag) data available, and it is unlikely that their genomes will be sequenced soon [42]. (For relevant public databases see dbEST of NCBI [43], The Gene Index Project (TGI) database [44], and the Taxonomically Broad EST DataBase (TBestDB)
[45]). When attempting to use available localization prediction tools on protein sequences conceptually translated from ESTs, we realized that prediction accuracy is generally very low. We tested the performance of seven state-of-the-art tools with proteins inferred from plant ESTs, and the overall accuracies were below 50\% (Table 1). This is not surprising, because these tools have been designed for full-length proteins and not for ESTs, which often represent only partial coding regions with an average length of $\sim 200$ residues. Further, ESTinferred proteins (referred to as EST-peptides from here on) may have an amino acid composition that differs from that of the corresponding full-length proteins. More importantly, EST-peptides often lack the N-terminal region of the corresponding proteins, which usually contains the targeting signal.

Finally BLAST, which we showed above to be unsuited for localization prediction of full-length proteins, is equally unsuited on EST-peptide data. Even at sequence identity levels above $90 \%$, the class-averaged accuracy for plant ESTs was below 75\% (Additional file 1 ). In practice, the accuracy would be even lower as EST projects often discover novel proteins that lack matches in databases. For example, in a large-scale protist EST project, more than $60 \%$ of ESTs could not find informative matches $[45,46]$. For the ESTs from such projects, the overall accuracy of localization prediction by BLAST would thus be less than $30 \%$.

We set out to develop a method that is tailored for predicting subcellular localization based on ESTs. As a test case we used plant data, which, as mentioned above, are more challenging than those from non-photosynthetic taxa. The methodology we developed can be readily applied to ESTs from any taxonomic groups, and the models we constructed can be easily retrained with sequences from a particular taxon of interest.

\section{Methods}

\section{Datasets}

We used two datasets: data from all plants to build and evaluate the localization prediction models (input data: EST-peptides), and Arabidopsis-only data to evaluate the combined prediction of ORFs and localization (input data: EST nucleotide sequences).

\section{Collection of protein sequences of experimentally verified} subcellular location

From SWISSPROT, we collected full-length Arabidopsis proteins localized in nine subcellular compartments: chloroplasts (chl), cytosol (cyt), endoplasmic reticulum (end), extracellular space (ext), mitochondria (mit), nucleus (nuc), peroxisomes (per), plasma membrane (pla), and vacuole (vac). Protein sequences were selected by the following criteria: 1 ) they are encoded by the nuclear genome; 2) their subcellular localization is 
Table 1 Performance of available tools and TESTLoc on plant EST-peptides ${ }^{1}$

\begin{tabular}{|c|c|c|c|c|c|c|c|c|c|c|c|c|c|c|c|c|c|c|}
\hline \multirow[t]{2}{*}{ Predictors } & \multicolumn{2}{|c|}{$\mathrm{chl}^{2}$} & \multicolumn{2}{|c|}{ cyt } & \multicolumn{2}{|c|}{ end } & \multicolumn{2}{|c|}{ ext } & \multicolumn{2}{|c|}{ mit } & \multicolumn{2}{|c|}{ nuc } & \multicolumn{2}{|c|}{ per } & \multicolumn{2}{|c|}{ pla } & \multicolumn{2}{|c|}{ vac } \\
\hline & SN & PPV & SN & PPV & SN & PPV & SN & PPV & SN & PPV & SN & PPV & SN & PPV & SN & PPV & SN & PPV \\
\hline TargetP & 19 & 62 & & & & & 29 & 25 & 18 & 44 & & & & & & & & \\
\hline BaCelLo & 61 & 69 & 50 & 28 & & & 60 & 32 & 9 & 90 & 88 & 39 & & & & & & \\
\hline Wolf-PSORT & 27 & 41 & 60 & 27 & 0 & 0 & 18 & 36 & 8 & 58 & 65 & 71 & 16 & 50 & 0 & 0 & 0 & 0 \\
\hline YLoc & 25 & 77 & 84 & 13 & 22 & 13 & 52 & 98 & 35 & 84 & 82 & 80 & 50 & 26 & 0 & 0 & 15 & 31 \\
\hline KnowPred & NA & NA & 71 & 23 & 0 & 0 & 23 & 36 & 61 & 49 & 86 & 40 & 58 & 54 & 0 & 0 & NA & NA \\
\hline MultiLoc2 & 14 & 81 & 84 & 12 & 9 & 8 & 44 & 48 & 18 & 37 & 48 & 67 & 67 & 10 & 0 & 0 & 45 & 54 \\
\hline$B L A S T^{3}$ & 76 & 97 & 77 & 62 & 64 & 100 & 81 & 95 & 57 & 87 & 77 & 96 & 25 & 60 & 0 & 0 & 0 & 0 \\
\hline TESTLoc & 99 & 99 & 50 & 88 & 20 & 20 & 71 & 98 & 86 & 71 & 75 & 96 & 50 & 21 & 30 & 5 & 63 & 100 \\
\hline
\end{tabular}

${ }^{1}$ Numbers in \%. Bold numbers are the result of the here described TESTLoc, which is tailored for ESTs. The results of full-length proteins are compiled in Additional file 8 .

${ }^{2}$ Abbreviations: chl, chloroplast; cyt, cytosol; end, endoplasmatic reticulum; ext, extracellular space; mit, mitochondrion; nuc, nucleus; per, peroxisome; pla, plasma membrane; vac, vacuole; SN, sensitivity; PPV, positive predictive value.

${ }^{3}$ Note that all test data have homologs in databases, which in practice is rarely the case; see text.

experimentally verified; and 3) the localization annotation is unambiguous (i.e., terms like "probable" or "possible" are absent from their subcellular localization annotation). These are strict criteria in order to avoid false positives in the dataset. No bias was detected as to the functional categories of proteins collected this way compared to other studies [47] (Additional file 3).

\section{Arabidopsis ESTs dataset}

The ESTs corresponding to the above Arabidopsis proteins were found via a similarity search by BLASTX in dbEST of GenBank, using a procedure illustrated in Additional file 4. When the aligned region of an EST was longer than $90 \%$ of its total length and the amino acid identity between the protein and the translated EST was $>90 \%$, we regarded the pair of EST and protein as belonging to the same gene. The selected ESTs were translated by EMBOSS Transeq [48] into amino acid sequences in the frame indicated by the BLASTX alignment. Sequence redundancy within the collected data was reduced by the tool CD-hit [49] so that no pair of sequences shares more than $60 \%$ identity. We obtained a dataset of 386 ESTs. Table 2 compiles the number of instances in each subcellular class.

\section{Plant ESTs dataset}

Using the same procedure as described above for Arabidopsis, we generated EST-peptides for all other plants with known localization (440 sequences). These combined with the above described Arabidopsis dataset constitute the plant dataset (826 sequences), which was

\section{Table 2 Number of EST-peptides used in this study}

\begin{tabular}{ccccccccccc}
\hline dataset & chl & cyt & end & ext & mit & nuc & per & pla & vac & total \\
\hline Arabidopsis & 97 & 53 & 5 & 9 & 167 & 41 & 5 & 4 & 5 & 386 \\
$\begin{array}{c}\text { Expanded plant } \\
\text { data }\end{array}$ & 679 & 122 & 11 & 48 & 309 & 260 & 12 & 7 & 29 & 1477 \\
& & & & & & & & & & \\
\end{tabular}

then used to build the expanded plant dataset as specified in the following.

\section{Expanded plant ESTs dataset}

Machine learning schemes perform better when trained with larger datasets. But in practice, the size of training data is often limited by their availability. We noticed that in our initial collection of EST-peptides, a number of proteins with known subcellular location were absent, because they have no corresponding EST sequences in public databases. To construct a training set with optimal coverage, the missing EST-peptides were substituted by artificial ones, generated by breaking up full-length proteins into overlapping pieces of $\sim 200$ residues. In this way, we almost tripled the size of training data. The procedure is described below.

The collected full-length protein sequences from plants were processed according to the following rules (Figure 1):

(1) When a sequence was shorter than 200 residues, it remained unchanged.

(2) When a sequence was 200 to 400 residues long, fragments of length ranging from 140 to 260 residues were generated from both the $\mathrm{N}$-terminus and $\mathrm{C}$-terminus. The range was based on a survey of the length distribution of ESTs, which revealed a mean of $\sim 600 \mathrm{nt}$ with a standard deviation of $\sim 180 \mathrm{nt}$. The $\mathrm{N}$-terminal fragment started within 80 residues from the first methionine, and the C-terminal fragment ended at the last amino acid. This simulated the nature of ESTs, which usually contain the complete $\mathrm{C}$-terminal, but lack the $\mathrm{N}$-terminal region.

(3) When a sequence was longer than 400 residues, an additional central fragment was generated starting anywhere downstream of the first 80 residues, but before the middle position of the original sequence. 


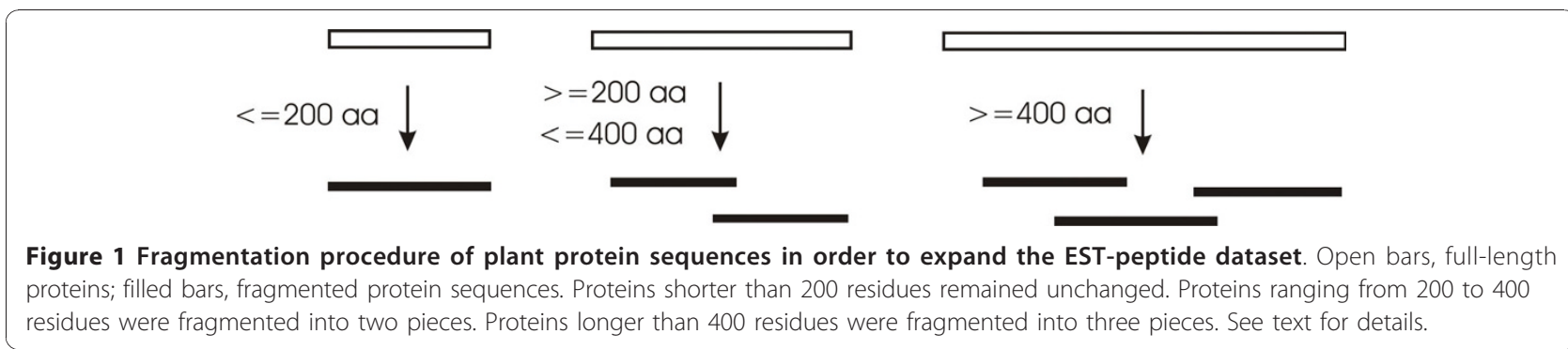

The fragmented protein sequences were combined with EST-derived peptides and clustered by CD-hit ( $\mathrm{Li}$ and Godzik, 2006) using a threshold of $60 \%$. The final dataset contains sequences from 41 species.

\section{Localization prediction by dedicated tools and BLAST}

We collected the best-performing localization prediction tools: TargetP, Protein Prowler, BaCelLo, Wolf-PSORT, YLoc, KnowPred, and MultiLoc2 (see Introduction), and tested their performance on the expanded plant dataset. The results of Wolf-POSRT and MultiLoc2 were obtained from a locally installed version. The prediction of YLoc was provided by its author. For the remaining tools, the results were obtained from their corresponding web-server.

To assess how BLAST performs for localization prediction of ESTs, the EST data were blasted against proteins in SWISSPROT, and the localization information of the second match (the first match is the same protein as the query ESTs, see data collection above) is transferred to the ESTs.

\section{Implementation of Support Vector Machine}

Features used to represent the peptide sequences for input of SVM

Physicochemical properties Physicochemical properties of amino acids in a protein can be represented by amino acid indices (AAindex, developed by the Amino Acid Index Database (http://www.genome.jp/dbget-bin/ show_man?aaindex)). The database currently contains 494 features for each amino acid (such as values of hydrophobicity, bulkiness, alpha-helix, turn, beta-sheet propensity, etc.). For each amino acid feature, its value was added up for the whole sequence, and was normalized by the sequence length. Subsequently, each ESTpeptide was converted into a 494-dimension vector.

Amino acid composition Six different types of amino acid composition were calculated. These include the frequency of individual amino acids ( $1^{\text {st }}$ order $)$, di-peptides $\left(2^{\text {nd }}\right.$ order $)$, tri-peptides $\left(3^{\text {rd }}\right.$ order $)$, tetra-peptides $\left(4^{\text {th }}\right.$ order), penta-peptides ( $5^{\text {th }}$ order), and hexa-peptides $\left(6^{\text {th }}\right.$ order) in the input sequence.

Grouped amino acid composition Amino acids were grouped according to their properties (Table 3 ). The alphabet of 20 amino acids was replaced by an alphabet of size eight (group C) or size ten (group D). Group C classified amino acids according to their chemical properties, which have shown good performance for localization prediction of full-length proteins [50]. Group D classified amino acids according to their structure [51]. After converting EST sequences using these new alphabets, the composition of amino acid groups was calculated from $1^{\text {st }}$ to $8^{\text {th }}$ order.

Gapped amino acid composition This feature represents the frequency of two amino acids (or amino acid groups) separated by $\mathrm{x}$ residues, $\mathrm{x}$ being the gap length. We experimented with gap lengths from 1 to 6 .

Table 3 Amino acids grouped according to their chemical properties

\begin{tabular}{|c|c|c|c|c|}
\hline \multicolumn{2}{|c|}{ Group C, chemical properties } & \multicolumn{3}{|c|}{ Group D, Devlin structural properties } \\
\hline Property & Amino acid & Superstructure & Structure & Amino acid \\
\hline Acidic & $D, E$ & Monoamino Moncarboxylic & & $G, A$ \\
\hline Basic & $\mathrm{H}, \mathrm{K}, \mathrm{R}$ & & Unsubstituted & $V, L, I$ \\
\hline Aromatic & $F, W, Y$ & & Heterocyclic & $P, F$ \\
\hline Small hydroxyl & $\mathrm{S}, \mathrm{T}$ & & Aromatic & $W, Y$ \\
\hline Sulphur containing & $C, M$ & & Thioether & M \\
\hline Aliphatic1 & $A, G, P$ & & Hydroxy & $S, T$ \\
\hline Aliphatic2 & $\mathrm{I}, \mathrm{L}, \mathrm{V}$ & & Mercapto & $C$ \\
\hline \multirow[t]{3}{*}{ Amide } & $\mathrm{N}, \mathrm{Q}$ & & Carboxamide & $N, Q$ \\
\hline & & Monamino, Dicarboxylic & & $D, E$ \\
\hline & & Diamino, Monocarboxylic & & $\mathrm{H}, \mathrm{K}, \mathrm{R}$ \\
\hline
\end{tabular}


Parameter selection and evaluation of SVM predictions In this study, we employed the SVM package LIBSVM [52], with the radial basis function (RBF) adopted as kernel function $\left(K\left(x_{i}, x_{j}\right)=\exp \left(-\gamma|| x_{i}-x_{j} \|^{2}\right), \gamma>0\right)$, which requires the selection of the kernel parameter $\gamma$, and the penalty parameter C. To obtain the optimal parameters and to evaluate the predictions, we performed a 10-fold cross validation scheme for SVM parameter ( $\mathrm{C}$ and $\gamma$ ) selection, followed by a 10-fold independent evaluation to assess the prediction performance (Figure 2). We first randomly divided the whole dataset into ten subsets of equal size. For each iteration of the ten rounds, nine subsets were combined to build SVM models, and the remaining subset was used for evaluation. The combined nine subsets were further subdivided into ten groups, whereof nine groups were combined and used to train SVM with given values for $\mathrm{C}$ and $\gamma$, while the remaining group was used to find the optimal combination of the two parameters. Finally, we assessed the performance of the selected $C$ and $\gamma$ values by using the evaluation data subset.

\section{Performance evaluation}

We calculated the overall accuracy for all classes combined, as well as the sensitivity (SN), specificity (SP), positive predictive value (PPV), and Matthews Correlation Coefficient (MCC) of each individual class as follows:

$$
\text { Overall Accuracy }(\mathrm{acc})=\frac{\sum_{\mathrm{i}=1}^{\mathrm{n}} \mathrm{TP} \mathrm{i}}{\sum_{\mathrm{i}=1}^{\mathrm{n}}(\mathrm{TP} \mathrm{i}+\mathrm{FNi})} * 100
$$

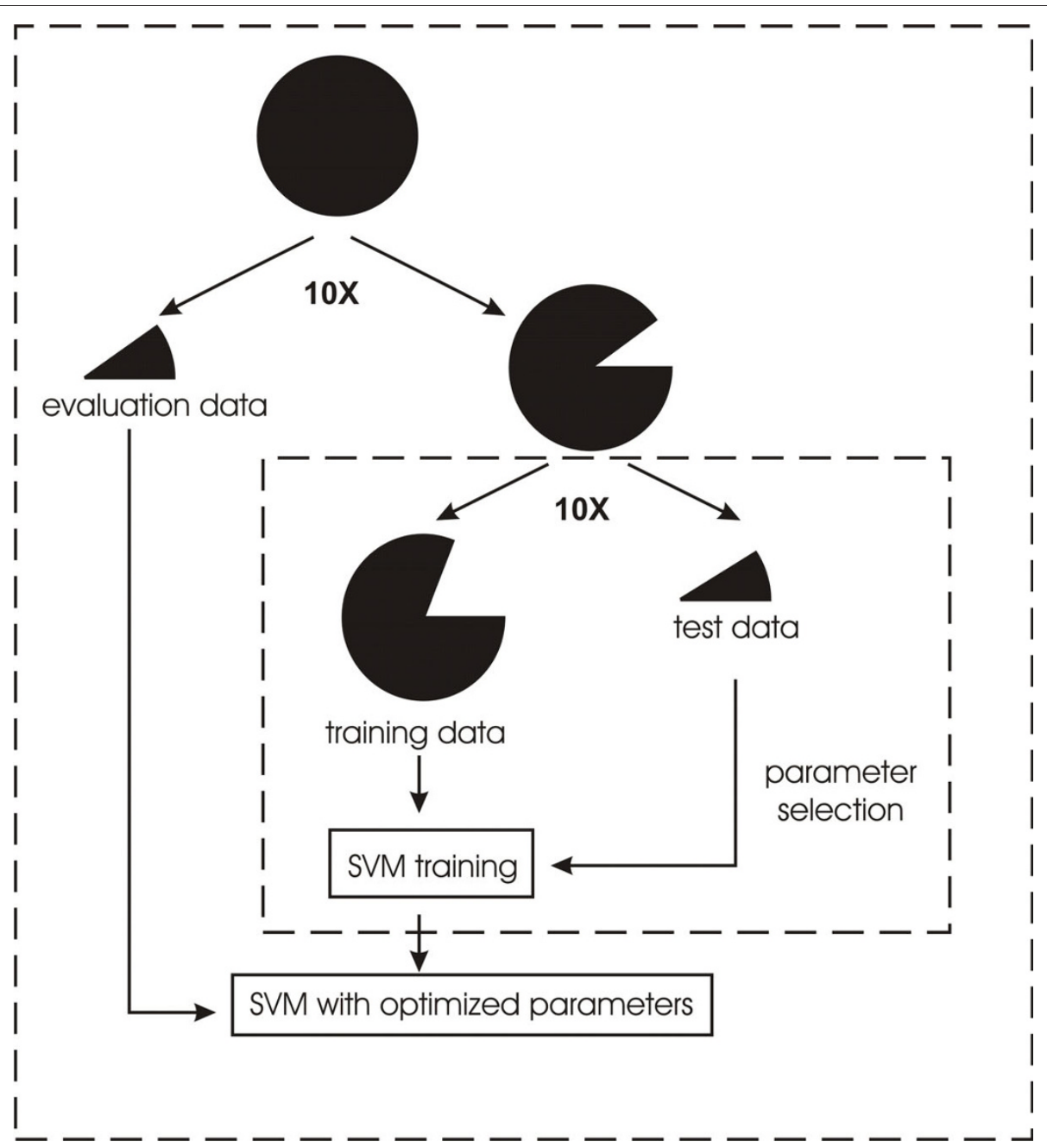

Figure 2 Training and evaluation of SVM predictors. The circle and pies indicate the dataset and portions thereof. The procedure in each dashed box was repeated ten times. The whole dataset was randomly divided into ten parts, with nine parts combined to construct the SVM model, and the remaining one to evaluate the model. The combined data for model construction were further divided randomly into ten subsets, in which nine subsets were combined to serve as training data, and the $10^{\text {th }}$ subset served as test data. See text for details. 
i: the i-th class; n: total number of classes

For each class i:

$$
\begin{aligned}
& \text { Sensitivity }(\mathrm{SNi})=\frac{\mathrm{TPi}}{\mathrm{TPi}+\mathrm{FNi}} * 100 \\
& \text { Specificity }(\mathrm{SPi})=\frac{\mathrm{TNi}}{\mathrm{TNi}+\mathrm{FPi}} * 100 \\
& \text { Positive predictive value }(\mathrm{PPVi})=\frac{\mathrm{TPi}^{\mathrm{TPi}+\mathrm{FPi}}}{} * 100 \\
& \text { Matthews Correlation Coefficient }(\mathrm{MCCi})= \\
& \frac{\mathrm{TPi}^{*} \mathrm{TNi}-\mathrm{FPi}{ }^{*} \mathrm{FNi}}{\sqrt{(\mathrm{TPi}+\mathrm{FPi})} *(\mathrm{TPi}+\mathrm{FNi}) *(\mathrm{TNi}+\mathrm{FPi}) *(\mathrm{TNi}+\mathrm{FNi})}
\end{aligned}
$$

TP: true positives; FP: false positives; TN: true negatives; FN: false negatives

\section{Open reading frame (ORF) prediction for ESTs}

Prot4EST [53] was used for the prediction of open reading frames (ORFs) in EST sequences. ESTs were first aligned with proteins from the NCBI non-redundant sequence database by BLASTX. The protein-EST alignment indicates the correct translation frame. For ESTs without significant BLAST matches, we used ESTScan [54] from within Prot4EST. ESTScan predicts ORFs based on a Hidden Markov Model (HMM) by recognizing the species-specific bias in hexanucleotide composition associated with coding and non-coding regions, and generates a matrix to represent this bias [54]. To generate the matrix, we trained ESTScan with all annotated Arabidopsis genomic and mRNA data collected from the European Molecular Biology Laboratory (EMBL) Nucleotide Sequence Database [55]. The mRNA data were mapped to genomic data in order to find the borders of coding/non-coding regions, which was needed to train the HMM. More details can be found in the publication describing ESTScan [54].

\section{Results}

We experimented with different sequence features to represent EST-peptides in SVM-based prediction of subcellular localization. As detailed in the Methods section, features included amino acid composition, grouped amino acid composition reflecting the physicochemical properties, gapped amino acid composition capturing the spatial context, as well as combinations thereof. The performance of the obtained prediction schemes varies considerably as shown below.
Performance of predictors based on individual features Among the 41 sequence features investigated (Additional file 5), the best performance was obtained by the SVM predictor exploiting the $4^{\text {th }}$ order amino acid composition (Figure 3; Table 4, scheme 1), with a MCC $>0.6$ for all large classes (nuclear, cytosolic, mitochondrial, and chloroplastic locations; for sensitivity and positive predictive value, see Additional file 6). A similarly good performance was observed with SVM predictors based on the $6^{\text {th }}$ order group-C amino acid composition and the $7^{\text {th }}$ order group-D amino acid composition (Additional file 5). Unexpectedly, sequence features such as gapped amino acid composition and physicochemical properties represented by AAindices did not yield satisfying results, with MCCs for most classes below 0.4 (Additional file 5). Three classes (plasma membrane, peroxisome and endoplasmic reticulum) are underrepresented, because data from these locations are still scarce. We included these classes because in practice, a query sequence could be from any of these locations. Were these locations left out, the predictor would inevitably misassign query sequences from these three classes. Yet, when these locations are included in the predictor, there is at least the possibility that the query sequence will be correctly assigned, even if the prediction accuracy may be low (20-50\%). Eventually, this shortcoming will be alleviated when more data from these locations become available.

\section{Performance of predictors based on combined features}

Previous studies showed that integration of multiple sequence features improves the performance of localization prediction $[31,32,34]$. We combined all the 41 sequence features described in the Methods section first by integrating attributes and second by integrating prediction results. To integrate attributes, the vectors of all sequence features were combined and used as input for SVM predictors. This type of integration yielded much lower performance than the best predictor based on a single feature (Table 4 , scheme 3 ).

Integration of prediction results from individual features was achieved by a two-layer SVM (Figure 4). The first layer consisted of SVM predictors based on a single sequence feature, yielding as output the probabilities of the query sequence to belong to each class. The outputs of all first-layer SVMs were combined and served as input for the second-layer SVM. Thus, each sequence was converted to a vector of size 369 (nine predictions for each of the 41 features). The two-layer SVM predictor showed similarly good performance as that based on the best single feature (Table 4, scheme 2).

In addition, we integrated the three features that yielded best performance ( $4^{\text {th }}$ order amino acid composition, $6^{\text {th }}$ order group-C composition and $7^{\text {th }}$ order group-D composition), as well as the predictions from 


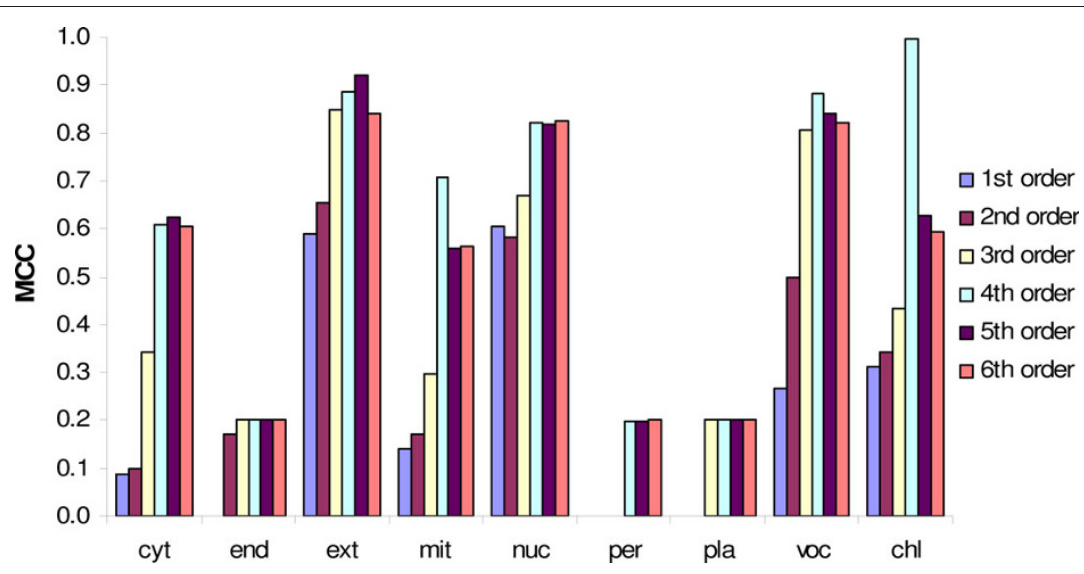

Figure 3 Independent evaluation of SVM predictors based on different representations of amino acid composition. The performance was assessed by the Matthews Correlation Coefficient (MCC). For most classes, the best MCC was obtained with the $4^{\text {th }}$ order amino acid composition (the frequency of tetra-peptides). Amino acid group- $C$ and group-D composition yielded similar results (see Additional file 5).

Table 4 Evaluation of results from top-ranking TESTLoc prediction schemes ${ }^{1}$

\begin{tabular}{|c|c|c|c|c|c|c|c|c|c|c|c|}
\hline & Prediction scheme & & chl & cyt & end & ext & mit & nuc & per & pla & vac \\
\hline \multirow[t]{15}{*}{ Expanded plant dataset } & $\begin{array}{l}\text { 1. Top-performing individual feature } \\
\text { ( } 4^{\text {th }} \text { order amino acid composition) }\end{array}$ & SN & $\begin{array}{l}99.9 \\
(0.44)\end{array}$ & $\begin{array}{c}\mathbf{5 3} \\
(12.2)\end{array}$ & $\begin{array}{c}20 \\
(42.2)\end{array}$ & $\begin{array}{c}83 \\
(17.7)\end{array}$ & $\begin{array}{c}88.4 \\
(7)\end{array}$ & $\begin{array}{l}82.7 \\
(4.9)\end{array}$ & $\begin{array}{c}20 \\
(42.2)\end{array}$ & $\begin{array}{c}20 \\
(42.2)\end{array}$ & $\begin{array}{c}80 \\
(23.3)\end{array}$ \\
\hline & & PPV & $\begin{array}{c}99.9 \\
(0.44)\end{array}$ & $\begin{array}{l}76.1 \\
(14.4)\end{array}$ & $\begin{array}{c}20 \\
(42.2)\end{array}$ & $\begin{array}{l}96.3 \\
(7.8)\end{array}$ & $\begin{array}{l}67.9 \\
(4.7)\end{array}$ & $\begin{array}{c}88 \\
(4.8)\end{array}$ & $\begin{array}{c}20 \\
(42.2)\end{array}$ & $\begin{array}{c}20 \\
(42.2)\end{array}$ & $\begin{array}{l}100 \\
(0)\end{array}$ \\
\hline & & MCC & $\begin{array}{c}0.99 \\
(0.01)\end{array}$ & $\begin{array}{c}0.61 \\
(0.14)\end{array}$ & $\begin{array}{c}0.2 \\
(0.42)\end{array}$ & $\begin{array}{l}0.88 \\
(0.1)\end{array}$ & $\begin{array}{c}0.7 \\
(0.07)\end{array}$ & $\begin{array}{l}0.82 \\
(0.04)\end{array}$ & $\begin{array}{c}0.2 \\
(0.42)\end{array}$ & $\begin{array}{c}0.2 \\
(0.42)\end{array}$ & $\begin{array}{l}0.88 \\
(0.14)\end{array}$ \\
\hline & $\begin{array}{l}\text { 2. Integration of predictions from } \\
\text { all sequence features }\end{array}$ & SN & $\begin{array}{l}100 \\
(0)\end{array}$ & $\begin{array}{l}45.5 \\
(8.55)\end{array}$ & $\begin{array}{c}20 \\
(42.2)\end{array}$ & $\begin{array}{l}69 \\
(12)\end{array}$ & $\begin{array}{l}86.1 \\
(10.8)\end{array}$ & $\begin{array}{l}78.1 \\
(8.3)\end{array}$ & $\begin{array}{c}40 \\
(51.6)\end{array}$ & $\begin{array}{c}30 \\
(48.3)\end{array}$ & $\begin{array}{l}63.3 \\
(24.6)\end{array}$ \\
\hline & & PPV & $\begin{array}{l}99.3 \\
(1.4)\end{array}$ & $\begin{array}{l}93.5 \\
(8.6)\end{array}$ & $\begin{array}{c}20 \\
(42.2)\end{array}$ & $\begin{array}{c}98 \\
(6.3)\end{array}$ & $\begin{array}{l}70.3 \\
(11.6)\end{array}$ & $\begin{array}{l}97.4 \\
(4.2)\end{array}$ & $\begin{array}{c}11.7 \\
(31.2)\end{array}$ & $\begin{array}{c}8.8 \\
(16.7)\end{array}$ & $\begin{array}{l}100 \\
(0)\end{array}$ \\
\hline & & MCC & $\begin{array}{c}0.99 \\
(0.01)\end{array}$ & $\begin{array}{c}0.63 \\
(0.08)\end{array}$ & $\begin{array}{c}0.2 \\
(0.42)\end{array}$ & $\begin{array}{c}0.81 \\
(0.07)\end{array}$ & $\begin{array}{c}0.7 \\
(0.05)\end{array}$ & $\begin{array}{l}0.85 \\
(0.05)\end{array}$ & $\begin{array}{c}0.16 \\
(0.31)\end{array}$ & $\begin{array}{l}0.15 \\
(0.26)\end{array}$ & $\begin{array}{c}0.78 \\
(0.16)\end{array}$ \\
\hline & $\begin{array}{l}\text { 3. Integration attributions of all } \\
\text { sequence features }\end{array}$ & SN & $\begin{array}{l}100 \\
(0)\end{array}$ & $\begin{array}{c}9.3 \\
(9.2)\end{array}$ & $\begin{array}{c}10 \\
(31.6)\end{array}$ & $\begin{array}{l}48.5 \\
(22.9)\end{array}$ & $\begin{array}{l}82.2 \\
(8.2)\end{array}$ & $\begin{array}{l}80 \\
(6.2)\end{array}$ & $\begin{array}{c}0 \\
(0)\end{array}$ & $\begin{array}{c}0 \\
(0)\end{array}$ & $\begin{array}{c}0 \\
(0)\end{array}$ \\
\hline & & PPV & $\begin{array}{l}100 \\
(0)\end{array}$ & $\begin{array}{l}28.8 \\
(31.8)\end{array}$ & $\begin{array}{c}10 \\
(31.6)\end{array}$ & $\begin{array}{l}77.8 \\
(22)\end{array}$ & $\begin{array}{l}53.1 \\
(3.4)\end{array}$ & $\begin{array}{l}76.2 \\
(8.5)\end{array}$ & $\begin{array}{c}0 \\
(0)\end{array}$ & $\begin{array}{c}0 \\
(0)\end{array}$ & $\begin{array}{c}0 \\
(0)\end{array}$ \\
\hline & & MCC & $\begin{array}{c}1 \\
(0)\end{array}$ & $\begin{array}{c}0.1 \\
(0.16)\end{array}$ & $\begin{array}{c}0.1 \\
(0.32)\end{array}$ & $\begin{array}{c}0.6 \\
(0.13)\end{array}$ & $\begin{array}{c}0.5 \\
(0.07)\end{array}$ & $\begin{array}{c}0.7 \\
(0.07)\end{array}$ & $\begin{array}{c}0 \\
(0)\end{array}$ & $\begin{array}{c}0 \\
(0)\end{array}$ & $\begin{array}{c}0 \\
(0)\end{array}$ \\
\hline & $\begin{array}{l}\text { 4. Integration of predictions from three } \\
\text { top-performing features }{ }^{2}\end{array}$ & SN & $\begin{array}{c}99.9 \\
(0.44)\end{array}$ & $\begin{array}{l}50.5 \\
(9.5)\end{array}$ & $\begin{array}{c}20 \\
(42.2)\end{array}$ & $\begin{array}{c}71 \\
(12)\end{array}$ & $\begin{array}{c}86.7 \\
(17.7)\end{array}$ & $\begin{array}{l}75.8 \\
(7.9)\end{array}$ & $\begin{array}{c}50 \\
(52.7)\end{array}$ & $\begin{array}{c}30 \\
(48.3)\end{array}$ & $\begin{array}{l}63.3 \\
(24.6)\end{array}$ \\
\hline & & PPV & $\begin{array}{l}99.7 \\
(0.6)\end{array}$ & $\begin{array}{l}88.4 \\
(9.2)\end{array}$ & $\begin{array}{c}20 \\
(42.2)\end{array}$ & $\begin{array}{l}98 \\
(6.3)\end{array}$ & $\begin{array}{l}71.2 \\
(12.4)\end{array}$ & $\begin{array}{l}96.1 \\
(4.6)\end{array}$ & $\begin{array}{l}21.6 \\
(41.4)\end{array}$ & $\begin{array}{c}5 \\
(8.1)\end{array}$ & $\begin{array}{l}100 \\
(0)\end{array}$ \\
\hline & & MCC & $\begin{array}{l}0.99 \\
(0.01)\end{array}$ & $\begin{array}{c}0.65 \\
(0.09)\end{array}$ & $\begin{array}{c}0.2 \\
(0.42)\end{array}$ & $\begin{array}{l}0.83 \\
(0.07)\end{array}$ & $\begin{array}{l}0.71 \\
(0.06)\end{array}$ & $\begin{array}{l}0.82 \\
(0.04)\end{array}$ & $\begin{array}{l}0.26 \\
(0.4)\end{array}$ & $\begin{array}{l}0.12 \\
(0.19)\end{array}$ & $\begin{array}{l}0.78 \\
(0.16)\end{array}$ \\
\hline & $\begin{array}{l}\text { 5. Integration of attributes from three } \\
\text { top-performing features }\end{array}$ & SN & $\begin{array}{l}94.4 \\
(2.2)\end{array}$ & $\begin{array}{c}52.2 \\
(12.7)\end{array}$ & $\begin{array}{c}20 \\
(42.2)\end{array}$ & $\begin{array}{c}75 \\
(17.2)\end{array}$ & $\begin{array}{l}84.2 \\
(5.8)\end{array}$ & $\begin{array}{l}77.7 \\
(4.7)\end{array}$ & $\begin{array}{c}20 \\
(42.2)\end{array}$ & $\begin{array}{c}20 \\
(42.2)\end{array}$ & $\begin{array}{l}76.7 \\
(22.5)\end{array}$ \\
\hline & & PPV & $\begin{array}{l}86.6 \\
(3.7)\end{array}$ & $\begin{array}{l}90.5 \\
(11)\end{array}$ & $\begin{array}{c}20 \\
(42.2)\end{array}$ & $\begin{array}{c}96 \\
(8.4)\end{array}$ & $\begin{array}{l}67.8 \\
(3.7)\end{array}$ & $\begin{array}{c}92 \\
(4.3)\end{array}$ & $\begin{array}{c}20 \\
(42.2)\end{array}$ & $\begin{array}{c}20 \\
(42.2)\end{array}$ & $\begin{array}{l}100 \\
(0)\end{array}$ \\
\hline & & MCC & $\begin{array}{l}0.8 \\
(0.05)\end{array}$ & $\begin{array}{l}0.66 \\
(0.1)\end{array}$ & $\begin{array}{c}0.2 \\
(0.42)\end{array}$ & $\begin{array}{l}0.84 \\
(0.1)\end{array}$ & $\begin{array}{l}0.68 \\
(0.05)\end{array}$ & $\begin{array}{l}0.81 \\
(0.04)\end{array}$ & $\begin{array}{c}0.2 \\
(0.42)\end{array}$ & $\begin{array}{c}0.2 \\
(0.42)\end{array}$ & $\begin{array}{l}0.86 \\
(0.14)\end{array}$ \\
\hline \multirow[t]{3}{*}{ Arabidopsis validation dataset } & $\begin{array}{l}\text { Integration of predictions from three } \\
\text { top-performing features }\end{array}$ & SN & 47.4 & 58.5 & 80 & 100 & 89.8 & 90.2 & 0 & 100 & 100 \\
\hline & & PPV & 90.6 & 86.1 & 100 & 100 & 68.2 & 100 & 100 & 100 & 100 \\
\hline & & MCC & 0.42 & 0.67 & 0.89 & 1 & 0.57 & 0.94 & 0 & 1 & 1 \\
\hline
\end{tabular}

${ }^{1}$ Numbers are the average of the 10 -fold test. Numbers in parenthesis are the standard deviation. Bold numbers indicate the best values for each metric (SN PPV, MCC) in each class of the expanded plant dataset. The values for SN and PPV are given in \%. MCC, Matthews Correlation Coefficient. For other abbreviations, see footnote to Table 1.

${ }^{2}$ The three features are $4^{\text {th }}$ order amino acid composition, $6^{\text {th }}$ order group-C amino acid composition and $7^{\text {th }}$ order group-D amino acid composition. 


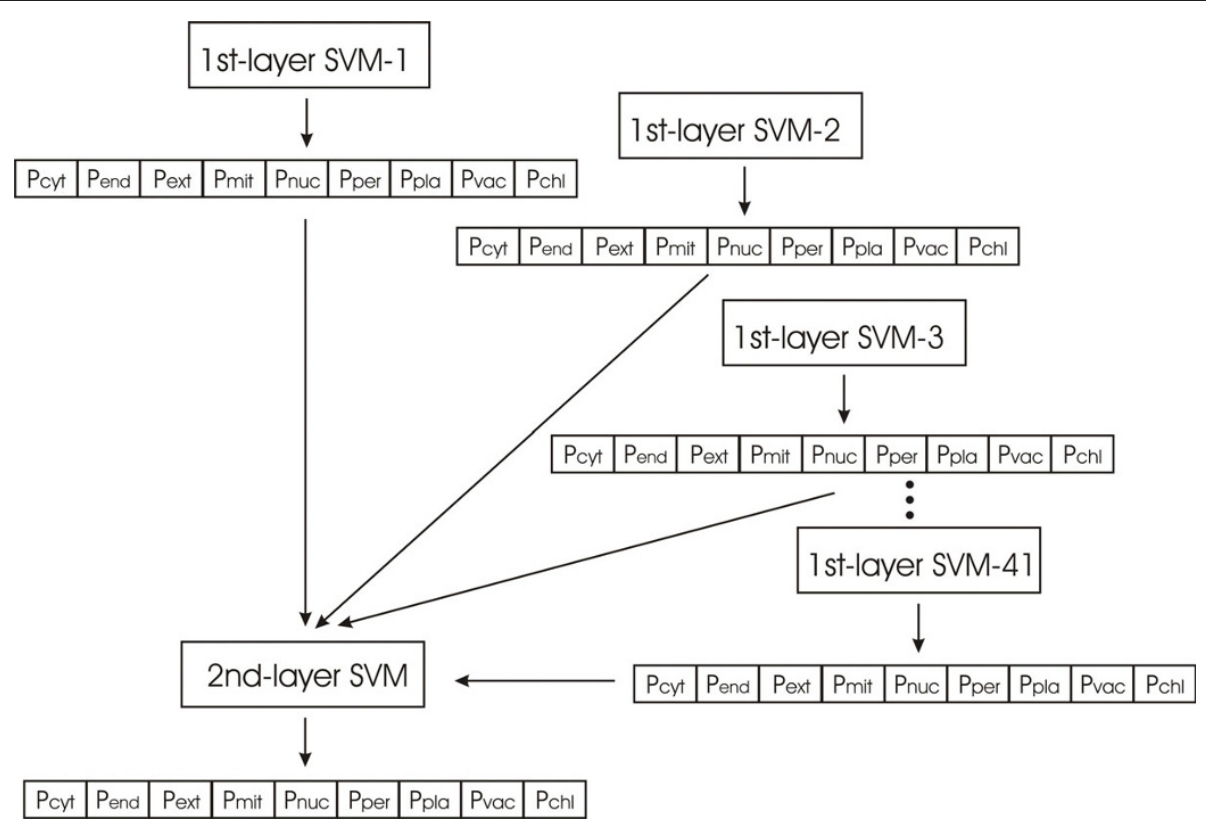

Figure 4 Integration of predictions from SVM models based on individual features. Each of the 41 SVM models built with single sequence features forms the first layer SVM and emits the probabilities for the query sequence to belong to the various classes. The probabilities are used as input for the second layer SVM.

these features. Again, these schemes showed a similar MCC as the predictors based on a single feature, but with lower sensitivity and higher positive predictive value for most classes (Table 4, scheme 4 and 5). Compared with the prediction from each of the three features individually, their integration often produced a much smaller rate of false positive predictions.

\section{ORF prediction from ESTs}

Another challenge in EST-peptide-based localization prediction is the correct translation from nucleotide sequence. Unlike genomic data, ESTs often lack start codon and 5'-UTR, which otherwise help to detect the correct open reading frame (ORF). In addition, ESTs are products of single-pass reads often containing low quality regions with sequencing errors that further complicate the task. Several tools have been developed for ORF identification in EST sequences $[3,54,56]$. We chose Prot4EST [53], which combines similarity-based and machinelearning-based prediction of ORFs. The scoring matrix specific to Arabidopsis was obtained by training ESTScan with Arabidopsis data, as described in the Methods section. The accuracy of ORF prediction, evaluated by the percentage of correctly identified start/stop positions of coding regions, was over 70\% (Additional file 7).

\section{Implementation of prediction methods and validation with Arabidopsis data}

We built a tool named TESTLoc that combines EST translation with localization prediction. TESTLoc has two components. The first takes EST nucleotide sequences as input and generates EST-peptides via the tool Prot4EST. The second takes EST-peptides as input and outputs the probability that the peptide is targeted to a given subcellular compartment. The current model predicts nine locations: cytosol, endoplasmic reticulum, extracellular space, mitochondria, nucleus, peroxisomes, plasma membrane, vacuole, and chloroplasts.

To evaluate the combined prediction of ORFs and subcellular localization, we tested the performance of TESTLoc with Arabidopsis ESTs that correspond to proteins of known localization. Sequences were represented by three best-performing features: $4^{\text {th }}$ order amino acid composition, $6^{\text {th }}$ order group-C amino acid composition, and $7^{\text {th }}$ order group-D amino acid composition. The resulting predictions showed high MCC values $(>0.6)$ for most classes (Table 4$)$.

TESTLoc can be downloaded and executed locally. The sequence features to use and how to combine them can be chosen by users via editing the configuration file. It should be emphasized that TESTLoc allows users to train new models with their own data, which facilitates the analysis of sequences from other taxonomic groups or individual species. (Should EST training data be scarce, artificial EST-peptides can be generated by breaking up full-length protein sequences as devised here.) Note that TESTLoc is designed for EST data, and should not be applied to full-length proteins (Additional file 8). 


\section{Discussion}

\section{Effects of various sequence features}

When experimenting with different kinds of $\mathrm{n}^{\text {th }}$ order amino acid composition, we observed a common trend: for the training data, the performance improved with increasing order until reaching a peak; for the evaluation data, at first the performance improved with the order, reached a peak, and then dropped again. This shows that higher-order composition made the scheme remember the instances in the training procedure, a phenomenon called overfitting. Therefore, we did not experiment with orders higher than six for ungrouped amino acid composition, and eight for grouped amino acid composition.

\section{Localization signals in partial sequences}

The sequence signals that guide the sorting of proteins into different subcellular compartments are not well understood. For a given compartment, more than one targeting signal seem to exist. Apparently, peroxisomal targeting involves at least three different signals, and mitochondrial targeting involves four $[57,58]$. Although characterized targeting signals are generally short $\mathrm{N}$ - or C-terminal peptide motifs, in many cases signals appear to be embedded in the central region of the protein. This explains why EST-peptides, which often lack the $\mathrm{N}$-terminal portion of a protein, are still informationrich enough for inferring subcellular localization, as we have demonstrated here.

In our study, tetra-peptides $\left(4^{\text {th }}\right.$ order amino acid composition) yielded the best performance. This is unexpected for a feature space two orders of magnitude larger $\left(20^{4}=160,000\right)$ than the sample size $(\sim 1,500$ sequences containing a total of $\sim 300,000$ tetra-peptides). Statistically, most tetra-peptides should be represented by only a few proteins, which should render machine learning rather ineffective. Alternatively, certain tetrapeptides may be over-represented in a given class, either due to a strong location-related signal or an artifact arising from redundancy in the dataset. To clarify the situation, we scrutinized the tetra-peptides present in our data (Additional file 9).

The size of the dataset allows for a total of 318,823 tetrapeptides. While the upper limit of distinct tetra-peptides (motifs) is 160,000, our particular dataset contains two third $(99,107)$. About one half $(45,883)$ of the occurring motifs are found in single classes and are present in only a few members of a given class $(<3 \%)$. Further, the motifs within a class do not show conserved sequence patterns. The complementary trend applies to the absence of tetrapeptides: no particular tetra-peptide is absent from only one class. In sum, there is no notable enrichment of, or bias against, certain tetra-peptide motifs in a class, nor any sign of a data redundancy artifact.
How then does this feature category achieve superior prediction performance? One possibility is that tetrapeptides bear targeting information in the form of more complex patterns such as nonadjacent correlation of multiple tetra-peptides, which we would not have recognized in our analysis.

\section{Conclusions}

Our results described here show that the SVM machine learning method, together with sequence features carefully chosen, predicts the subcellular location of ESTderived proteins with high accuracy, thus filling the need for a tool tailored to EST data. We implemented TESTLoc as a fully automated pipeline combining ESTORF prediction and localization prediction. This tool opens new avenues for systematic analysis of EST data from any eukaryote including challenging photosynthetic taxa such as plants.

\section{Additional material}

Additional file 1: Influence of sequence similarity on the accuracy
of localization prediction by TESTLoc and BLAST.
Additional file 2: List of available subcellular localization prediction
methods.
Additional file 3: Gene Ontology (GO) term of proteins from the
plant dataset used in this study.
Additional file 4: Selection of Arabidopsis ESTs corresponding to
proteins of known localization.
Additional file 5: Performance of predicting subcellular localization
of EST-derived proteins, based on each sequence feature (including
amino acid composition, grouped amino acid composition, gapped
amino acid composition, and AAindex).
Additional file 6: The independent evaluation of SVM predictors
based on different representations of amino acid composition,
measured with sensitivity and positive predictive value.
Additional file 7: Accuracy of ESTScan for the prediction of start/
stop positions of coding regions in EST sequences.
Additional file 8: Comparison of prediction performance of
available tools and TESTLoc on full-length plant protein sequences.
Additional file 9: Tetra-peptides present in the expanded plant ESTs
data.

\section{Acknowledgements}

We would like to thank Geneviève Galarneau for programming parts of TESTLOC and evaluating the accuracy of ORF prediction, and JeanFrançois Théroux for implementing gapped amino acid composition. Both projects were conducted in the context of a research internship course of the bioinformatics bachelor program at the Université de Montréal. We also thank the author of YLoc for conducting the prediction on our data. This work was supported by the Canadian Institute for Advanced Research (CIFAR). YQS received a stipend from the Canadian Institute for Health Research (CIHR) Strategic Training Grant in Bioinformatics.

\section{Authors' contributions}

YQS developed and implemented the methods. GB conceived of the study, participated in its design, and supervised the process. YQS drafted the manuscript. Both authors read and approved the final manuscript. 
Received: 1 July 2010 Accepted: 15 November 2010

Published: 15 November 2010

\section{References}

1. Huh WK, Falvo JV, Gerke LC, Carroll AS, Howson RW, Weissman JS, O'Shea EK: Global analysis of protein localization in budding yeast. Nature 2003, 425(6959):686-691.

2. Kumar A, Agarwal S, Heyman JA, Matson S, Heidtman M, Piccirillo S, Umansky L, Drawid A, Jansen R, Liu Y, et al: Subcellular localization of the yeast proteome. Genes Dev 2002, 16(6):707-719.

3. Barbe L, Lundberg E, Oksvold P, Stenius A, Lewin E, Bjorling E, Asplund A, Ponten $\mathrm{F}$, Brismar $\mathrm{H}$, Uhlen $\mathrm{M}$, et al: Toward a confocal subcellular atlas of the human proteome. Mol Cell Proteomics 2008, 7(3):499-508

4. Lascaris R, Bussemaker HJ, Boorsma A, Piper M, van der Spek H, Grivell L, Blom J: Hap4p overexpression in glucose-grown Saccharomyces cerevisiae induces cells to enter a novel metabolic state. Genome Biol 2003, 4(1):R3.

5. Yuan HM, Li KL, Ni RJ, Guo WD, Shen Z, Yang CP, Wang BC, Liu GF, Guo $\mathrm{CH}$, Jiang J: A systemic proteomic analysis of Populus chloroplast by using shotgun method. Mol Biol Rep 2010.

6. Li J, Cai T, Wu P, Cui Z, Chen X, Hou J, Xie Z, Xue P, Shi L, Liu P, et al: Proteomic analysis of mitochondria from Caenorhabditis elegans. Proteomics 2009, 9(19):4539-4553

7. Altschul SF, Madden TL, Schaffer AA, Zhang J, Zhang Z, Miller W, Lipman DJ: Gapped BLAST and PSI-BLAST: a new generation of protein database search programs. Nucleic Acids Res 1997, 25(17):3389-3402.

8. Nair R, Rost B: Sequence conserved for subcellular localization. Protein Sci 2002, 11(12):2836-2847.

9. Shen $Y Q$, Burger G: Plasticity of a key metabolic pathway in fungi. Funct Integr Genomics 2009, 9(2):145-151.

10. Shatkay H, Hoglund A, Brady S, Blum T, Donnes P, Kohlbacher O: SherLoc: high-accuracy prediction of protein subcellular localization by integrating text and protein sequence data. Bioinformatics 2007, 23(11):1410-1417.

11. Lu Z, Szafron D, Greiner R, Lu P, Wishart DS, Poulin B, Anvik J, Macdonell C, Eisner R: Predicting subcellular localization of proteins using machinelearned classifiers. Bioinformatics 2004, 20(4):547-556.

12. Nair R, Rost B: Inferring sub-cellular localization through automated lexical analysis. Bioinformatics 2002, 18(Suppl 1):S78-86.

13. Guda C, Subramaniam S: pTARGET [corrected] a new method for predicting protein subcellular localization in eukaryotes. Bioinformatics 2005, 21(21):3963-3969.

14. Scott MS, Thomas DY, Hallett MT: Predicting subcellular localization via protein motif co-occurrence. Genome Res 2004, 14(10A):1957-1966.

15. Claros MG, Vincens P: Computational method to predict mitochondrially imported proteins and their targeting sequences. Eur J Biochem 1996, 241(3):779-786.

16. Emanuelsson $\mathrm{O}$, Nielsen $\mathrm{H}$, Brunak S, von Heijne G: Predicting subcellular localization of proteins based on their $\mathrm{N}$-terminal amino acid sequence. $\mathrm{J} \mathrm{Mol} \mathrm{Biol} \mathrm{2000,} \mathrm{300(4):1005-1016}$

17. Bannai H, Tamada Y, Maruyama O, Nakai K, Miyano S: Extensive feature detection of $\mathrm{N}$-terminal protein sorting signals. Bioinformatics 2002, 18(2):298-305

18. Boden M, Hawkins J: Prediction of subcellular localization using sequence-biased recurrent networks. Bioinformatics 2005, 21(10):2279-2286.

19. Small I, Peeters N, Legeai F, Lurin C: Predotar: A tool for rapidly screening proteomes for $\mathrm{N}$-terminal targeting sequences. Proteomics 2004, 4(6):1581-1590.

20. Reinhardt A, Hubbard T: Using neural networks for prediction of the subcellular location of proteins. Nucleic Acids Res 1998, 26(9):2230-2236

21. Hua S, Sun Z: Support vector machine approach for protein subcellular localization prediction. Bioinformatics 2001, 17(8):721-728.

22. Huang Y, Li Y: Prediction of protein subcellular locations using fuzzy kNN method. Bioinformatics 2004, 20(1):21-28.

23. Chou KC: Prediction of protein cellular attributes using pseudo-amino acid composition. Proteins 2001, 43(3):246-255

24. Chou KC, Cai YD: Using functional domain composition and support vector machines for prediction of protein subcellular location. J Biol Chem 2002, 277(48):45765-45769
25. Park KJ, Kanehisa M: Prediction of protein subcellular locations by support vector machines using compositions of amino acids and amino acid pairs. Bioinformatics 2003, 19(13):1656-1663.

26. Sarda D, Chua GH, Li KB, Krishnan A: pSLIP: SVM based protein subcellular localization prediction using multiple physicochemical properties. BMC Bioinformatics 2005, 6:152.

27. Guda C, Fahy E, Subramaniam S: MITOPRED: a genome-scale method for prediction of nucleus-encoded mitochondrial proteins. Bioinformatics 2004, 20(11):1785-1794.

28. Bhasin M, Raghava GP: ESLpred: SVM-based method for subcellular localization of eukaryotic proteins using dipeptide composition and PSIBLAST. Nucleic Acids Res 2004, 32 Web Server: W414-419.

29. Nair R, Rost B: Mimicking cellular sorting improves prediction of subcellular localization. J Mol Biol 2005, 348(1):85-100.

30. Xie D, Li A, Wang M, Fan Z, Feng H: LOCSVMPSI: a web server for subcellular localization of eukaryotic proteins using SVM and profile of PSI-BLAST. Nucleic Acids Res 2005, , 33 Web Server: W105-110.

31. Yu CS, Lin CJ, Hwang JK: Predicting subcellular localization of proteins for Gram-negative bacteria by support vector machines based on n-peptide compositions. Protein Sci 2004, 13(5):1402-1406.

32. Blum T, Briesemeister S, Kohlbacher O: MultiLoc2: integrating phylogeny and Gene Ontology terms improves subcellular protein localization prediction. BMC Bioinformatics 2009, 10(1):274

33. Kaundal R, Saini R, Zhao PX: Combining machine learning and homologybased approaches to accurately predict subcellular localization in Arabidopsis. Plant Physiol 2010, 154(1):36-54

34. Shen YQ, Burger G: 'Unite and conquer': enhanced prediction of protein subcellular localization by integrating multiple specialized tools. BMC Bioinformatics 2007, 8:420.

35. Liu J, Kang S, Tang C, Ellis LB, Li T: Meta-prediction of protein subcellular localization with reduced voting. Nucleic Acids Res 2007, 35(15):e96.

36. Assfalg J, Gong J, Kriegel HP, Pryakhin A, Wei T, Zimek A: Supervised ensembles of prediction methods for subcellular localization. J Bioinform Comput Biol 2009, 7(2):269-285.

37. Casadio R, Martelli PL, Pierleoni A: The prediction of protein subcellular localization from sequence: a shortcut to functional genome annotation. Brief Funct Genomic Proteomic 2008, 7(1):63-73.

38. Briesemeister $\mathrm{S}$, Rahnenfuhrer J, Kohlbacher O: Going from where to whyinterpretable prediction of protein subcellular localization. Bioinformatics 2010, 26(9):1232-1238.

39. Pierleoni A, Martelli PL, Fariselli P, Casadio R: BaCelLo: a balanced subcellular localization predictor. Bioinformatics 2006, 22(14):e408-416.

40. Horton P, Park KJ, Obayashi T, Fujita N, Harada H, Adams-Collier CJ, Nakai K: WoLF PSORT: protein localization predictor. Nucleic Acids Res 2007, , 35 Web Server: W585-587.

41. Lin HN, Chen CT, Sung TY, Ho SY, Hsu WL: Protein subcellular localization prediction of eukaryotes using a knowledge-based approach. BMC Bioinformatics 2009, 10(Suppl 15):S8.

42. Parkinson J, Blaxter M: Expressed sequence tags: an overview. Methods Mol Biol 2009, 533:1-12

43. Boguski MS, Lowe TM, Tolstoshev CM: dbEST-database for "expressed sequence tags". Nat Genet 1993, 4(4):332-333.

44. Lee Y, Tsai J, Sunkara S, Karamycheva S, Pertea G, Sultana R, Antonescu V, Chan A, Cheung F, Quackenbush J: The TIGR Gene Indices: clustering and assembling EST and known genes and integration with eukaryotic genomes. Nucleic Acids Res 2005, 33 Database: D71-74

45. O'Brien EA, Koski LB, Zhang Y, Yang L, Wang E, Gray MW, Burger G, Lang BF: TBestDB: a taxonomically broad database of expressed sequence tags (ESTs). Nucleic Acids Res 2007, 35 Database: D445-451.

46. Koski LB, Gray MW, Lang BF, Burger G: AutoFACT: an automatic functional annotation and classification tool. BMC Bioinformatics 2005, 6:151.

47. Baerenfaller K, Grossmann J, Grobei MA, Hull R, Hirsch-Hoffmann M, Yalovsky S, Zimmermann P, Grossniklaus U, Gruissem W, Baginsky S: Genome-scale proteomics reveals Arabidopsis thaliana gene models and proteome dynamics. Science 2008, 320(5878):938-941.

48. Rice P, Longden I, Bleasby A: EMBOSS: the European Molecular Biology Open Software Suite. Trends Genet 2000, 16(6):276-277.

49. Li W, Godzik A: Cd-hit: a fast program for clustering and comparing large sets of protein or nucleotide sequences. Bioinformatics 2006, 22(13):1658-1659. 
50. Yu CS, Chen YC, Lu CH, Hwang JK: Prediction of protein subcellular localization. Proteins 2006, 64(3):643-651.

51. Devlin TM: The Textbook of Biochemistry. New York: Wiley-Liss Inc; 3 1992.

52. Fan RE, Chen $\mathrm{PH}$, Lin $\mathrm{CJ}$ : Working set selection using the second order information for training SVM. Journal of Machine Learning Research 2005 6:1889-1918.

53. Wasmuth JD, Blaxter ML: prot4EST: translating expressed sequence tags from neglected genomes. BMC Bioinformatics 2004, 5:187.

54. Iseli C, Jongeneel CV, Bucher P: ESTScan: a program for detecting, evaluating, and reconstructing potential coding regions in EST sequences. Proc Int Conf Intell Syst Mol Biol 1999, 138-148.

55. Kanz C, Aldebert P, Althorpe N, Baker W, Baldwin A, Bates K, Browne P, van den Broek A, Castro M, Cochrane G, et al: The EMBL Nucleotide Sequence Database. Nucleic Acids Res 2005, , 33 Database: D29-33.

56. Hatzigeorgiou AG, Fiziev P, Reczko M: DIANA-EST: a statistical analysis. Bioinformatics 2001, 17(10):913-919.

57. Chacinska A, Koehler CM, Milenkovic D, Lithgow T, Pfanner N: Importing mitochondrial proteins: machineries and mechanisms. Cell 2009, 138(4):628-644.

58. Platta HW, Erdmann R: The peroxisomal protein import machinery. FEBS Lett 2007, 581(15):2811-2819.

doi:10.1186/1471-2105-11-563

Cite this article as: Shen and Burger: TESTLoc: protein subcellular localization prediction from EST data. BMC Bioinformatics 2010 11:563.

\section{Submit your next manuscript to BioMed Central} and take full advantage of:

- Convenient online submission

- Thorough peer review

- No space constraints or color figure charges

- Immediate publication on acceptance

- Inclusion in PubMed, CAS, Scopus and Google Scholar

- Research which is freely available for redistribution

Submit your manuscript at www.biomedcentral.com/submit 